\title{
From the Observation of UHECR Radio Signal in [1-200] MHz to the Composition: CODALEMA and EXTASIS Status Report
}

\author{
Antony ESCUDIE*1, D. Charrier ${ }^{1,3}$, R. Dallier ${ }^{1,3}$, D. García-Fernández ${ }^{1}$, A. \\ Lecacheux $^{2}$, L. Martin ${ }^{1,3}$ and B. Revenu ${ }^{1,3}$ \\ ${ }^{1}$ SUBATECH, Institut Mines-Telecom Atlantique - CNRS/IN2P3 - Universite de Nantes, \\ Nantes, France \\ 2 CNRS-Observatoire de Paris, Meudon, France \\ ${ }^{3}$ Unite Scientifique de Nancay, Observatoire de Paris, CNRS, PSL, UO/OSUC, Nancay, France \\ E-mail: antony.escudieesubatech.in2p3.fr
}

\begin{abstract}
Over the years, significant efforts have been devoted to the understanding of the radio emission of extensive air shower (EAS) in the range [20-80] MHz but, despite some studies led until the nineties, the [1-10] MHz band has remained unused for nearly 30 years. The EXTASIS experiment, located within the radio astronomy observatory of Nançay and supported by the CODALEMA instrument, aims to reinvestigate the [1-10] $\mathrm{MHz}$ band, and to study the so-called "Sudden Death" contribution, the expected radiation electric field created by the particles that are stopped upon arrival to the ground. We present the instrumental setup and the results obtained with EXTASIS.

Moreover, CODALEMA has demonstrated that in [20-80] MHz, the commonly used band, the electric field profile of extensive air showers is well understood, and contains all the information needed for the reconstruction of extensive air showers. An analysis pipeline was developed to set up an online reconstruction of the primary cosmic ray properties. Combining the antenna and acquisition system capabilities, CODALEMA extended the observation window up to 200 $\mathrm{MHz}$, permitting to improve the quality of the reconstruction of the primary cosmic ray properties thanks to a fine calibration procedure. We present the results obtained on a large batch of cosmic ray events, notably a first estimate of their mass dependence with energy.
\end{abstract}

36th International Cosmic Ray Conference -ICRC2019-

July 24th - August 1st, 2019

Madison, WI, U.S.A.

\footnotetext{
* Speaker.
} 


\section{Introduction}

A coherent radio emission is emitted during the development of extensive air showers (EAS) through the transverse charged current variation induced by the geomagnetic field, and the charge excess mechanism $[1,2,3,4]$. The resulting electric field can be detected by large bandwidth antennas and fast acquisition systems. The observations are commonly carried out in the $[20-80] \mathrm{MHz}$ range (noted MF in the following, for Medium Frequencies) by experiments such as AERA [5] or LOFAR [6]. Thanks to its design, CODALEMA operates in the [20-200] MHz band, referred as EMF in the following for Extended Medium Frequencies. Moreover, the EXTASIS experiment [7] hosted by CODALEMA, dedicated to resume the low frequency (LF) studies made in the 70's and up to the 90's, permits to widen the frequency range, and to demonstrate for the first time that the study of atmospheric showers is possible continuously from 1 to $200 \mathrm{MHz}$ in a continuous way, making it possible to scan different zones and processes of their development. The goal of the study of cosmic rays is the reconstruction of their parameters, especially their mass and energy, primordial to understand the mechanisms of acceleration of cosmic rays, their propagation and their sources. These characteristics can be estimated by comparing the electric field detected at ground level to a large catalogue predictions obtained from detailed simulations.

\section{Instrumental setup}

CODALEMA is installed since 2002 at the Nançay Radioastronomy Observatory. It is one of the pioneering experiments that have participated in the rebirth of radio detection of cosmic rays. Over the years, the experiment has seen the development of a large collection of detectors, intended to study the properties of the radio emission associated with cosmic ray induced air showers in the energy range from $10^{16}$ to $10^{18} \mathrm{eV}$. In its current version, CODALEMA consists of a square array $\left(0.4 \times 0.4 \mathrm{~km}^{2}\right)$ of 13 scintillators (surface detector); a set of 57 so-called "autonomous" crossed dipoles (hereafter noted SA for standalone antennas), synchronized by GPS dating and operating in the EMF band, distributed over $1.1 \mathrm{~km}^{2}$; a so-called "Compact Array" (here after noted CA) of 10 cross-polarized antennas arranged in a star shape

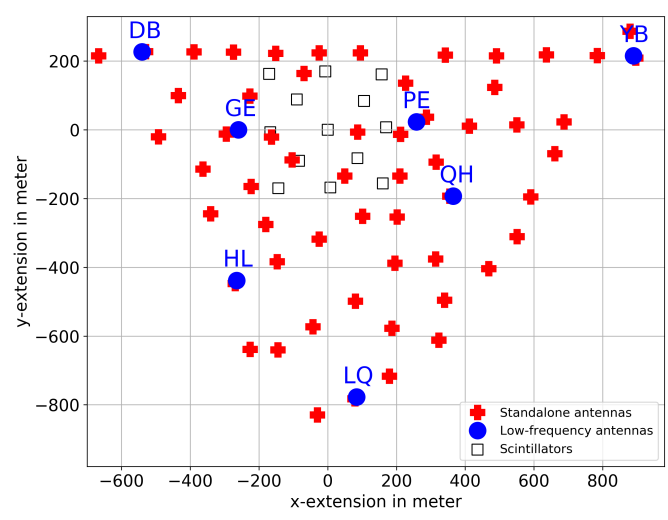

Figure 1: Area experiment at the Nançay Radioastronomy Observatory [7]. Red crosses represent the 57 standalone antennas of CODALEMA, black squares the 13 scintillators, the blue points represent the $7 \mathrm{LF}$ antennas. The Compact Array is not shown. of $150 \mathrm{~m}$ extension and whose signal acquisition (in the MF band) is triggered by the particle detector. CODALEMA is today the support infrastructure of EXTASIS [7], an array of 7 low-frequency antennas also triggered by the particle detector. The LF antenna locations have been chosen to cover the overall Nançay Radioastronomy Observatory area and to have a nearby MF autonomous station (see figure 1)). 


\section{Low-frequency observation - EXTASIS results}

The frequency band below $20 \mathrm{MHz}$ has remained unused for cosmic ray induced transient detection for almost 20 years. It has been reported by some pioneering experiments ([7] and references herein) that EAS emit a strong electric field and that there is an evidence of a large increase of the radio pulse amplitude with decreasing frequencies. We have re-investigated the $[1.7-3.7] \mathrm{MHz}$ band with the EXTASIS experiment and observed 25 air showers over $\sim 2$ years of observation, detected in coincidence by CODALEMA and EXTASIS, and correlated with cosmic-ray events. We have estimated a detection threshold of $23 \pm 4 \mu \mathrm{V} \cdot \mathrm{m}^{-1}$ from comparisons with detailed SELFAS3 $[8,9,10]$ (simulation code of the radio signal of cosmic-ray-induced air showers, using of a fine description of the atmosphere and taking into account the near field effects) simulations, and we have confirmed that the detection range at LF is larger than in the classical $[30-80] \mathrm{MHz}$ band. However, we have demonstrated that the harsh atmospheric noise conditions hamper the detection at LF. The low rate of detection in this band is mainly due to the atmospheric noise, which remains in the best case one order of magnitude higher than the amplitude of the signal that we want to detect. We have also highlighted a strong correlation of the LF signal observation with the atmospheric electric field. Concerning the so-called "sudden death phenomenon" (SDP) contribution (the expected electric field radiated by the shower front when hitting ground level), no sign of such signal has been observed by EXTASIS. It has not been yet detected at Nançay and we have shown that its probability of detection is favored for high altitude sites such as Auger [7].

\section{Calibration of the radio detectors of CODALEMA}

In the past, numerous disagreements have been reported on the amplitudes of the electric fields detected for a given energy [11], notably due to the difficulty to calibrate the radio detectors used at these times. In order to be as precise as possible on the measurement of the amplitudes, and thus on the estimation of the properties of the primary cosmic ray, a calibration of the radio detectors is mandatory. We aim to cross calibrate our radio instruments (the SA and CA antennas) using the Galactic radio emission. In the following, we only deal with the calibration of the SA (the same method has been applied on the CA antennas). The SA are equipped with a software trigger which is produced at regular intervals over the full autonomous station array and used to produce calibration data. These data contain mostly background noise: when the external disturbances are absent, only the galactic radio emission component remains, which can be used as a reference to cross calibrate our antennas. We used the Global Sky Model [12] for the temperature of the galactic radio emission. To calculate the contribution of each part of the sky, we have to convolve the skymap by the lobe of the antenna as a function of the Local Sidereal Time (LST). Then, we obtain a simulation of the measured signal at a given LST by summing each pixels of the convoluted skymap. Data were binned in LST-bins of 16 minutes. The result obtained is called a transit curve, that can be compared to the actual data. Figure 2 present the $\langle R M S\rangle$ (noted rms in lowercase in figures to lighten the notations) of the data as a function of LST. The transit curves are stackable, meaning that the source of the emission is the same for all the antenna array. The behaviour of the $\langle R M S\rangle$ of the data is similar for all the antennas over LST, and only an offset along the y-axis is present, that a proposer calibration should remove.

Figure 3 presents the predictions of the measured Galactic radio emission obtained with NEC4, for $50 \mathrm{MHz}$ (left) and for $150 \mathrm{MHz}$ (right) for the EW polarization. There is a good agreement 

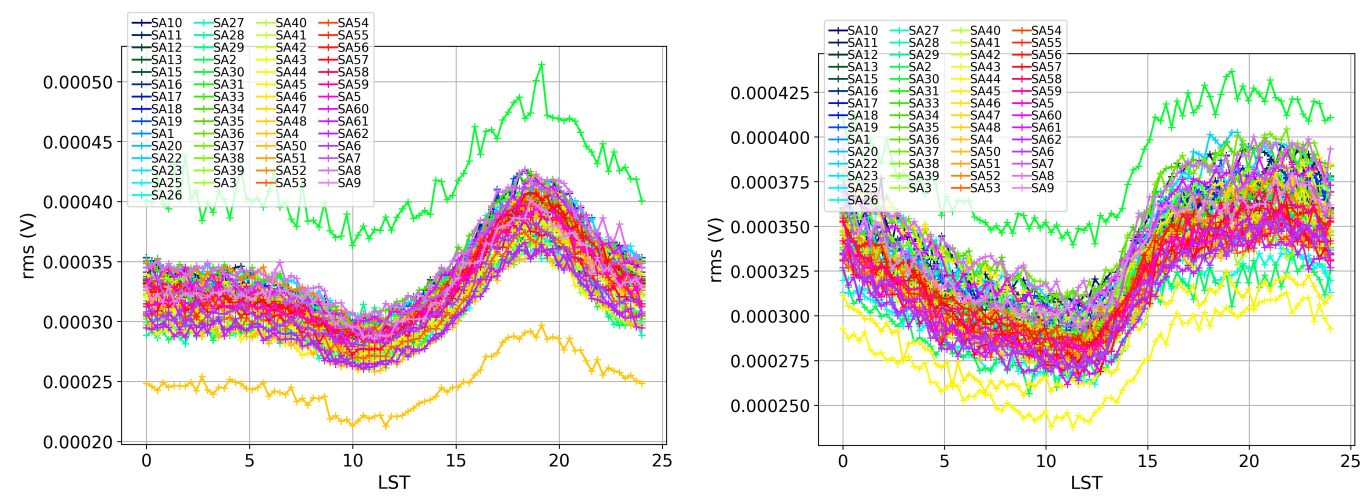

Figure 2: The RMS of the 1W data as a fucntion of Local Sidereal Time (LST) for the EW (left) and NS (right) polarizations at $50 \mathrm{MHz}$ (sub-bands of $10 \mathrm{MHz}$ width) and for an arbitrary month. For each LST bin, the $\langle R M S\rangle$ is calculated from the distribution of the RMS values of the background noise. Outliers in green and yellow are antennas with an electrical problem and come out of the range.

between the prediction made with NEC4 (blue curve) and the data at $50 \mathrm{MHz}$ and especially at $150 \mathrm{MHz}$ where the overall radiation is 10 times smaller than at $50 \mathrm{MHz}$, making us confident in the mastering of instrumental response.
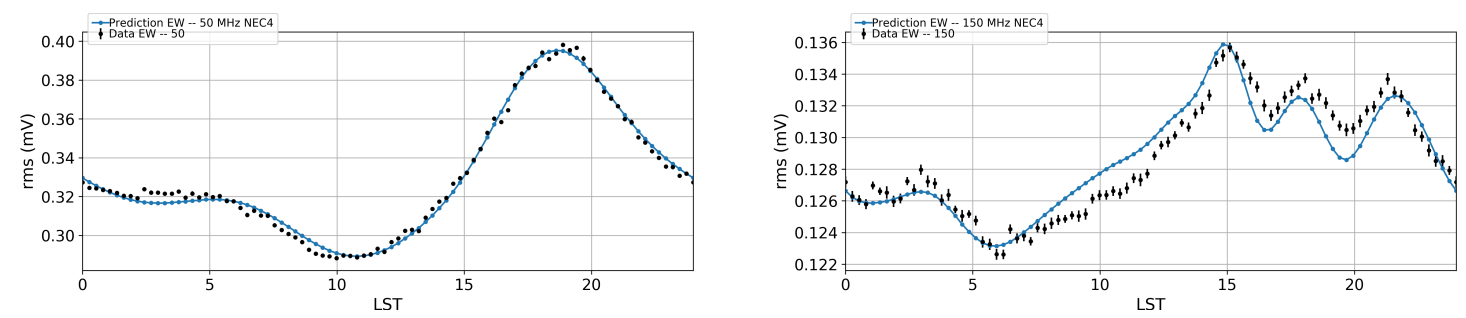

Figure 3: Left: $50 \mathrm{MHz}$-predictions of transit curve of the EW polarization fitted on data (black points). Right: same as left at $150 \mathrm{MHz}$.

The calculation of the calibration coefficients is made for all frequencies with a step of $1 \mathrm{MHz}$ and all antennas, month by month to take into account a seasonal variation observed in our data. Figure 4 presents the results obtained after the cross calibration of the $\mathrm{SA}$, at $50 \mathrm{MHz}$ for an arbitrary month, for the EW (left) and NS (right) polarizations, to be compared with figure 2. After the cross calibration, the dispersion over the whole antenna array stands in around $\sim 6 \%$.

In other words, at $50 \mathrm{MHz}$ and for the considered month, all the SA signals are now contained within a $20 \mu \mathrm{V}$ wide $\langle R M S\rangle$ interval. For a typical event, the RMS of the recorded signal of one antenna is equal to $\sim 700 \mu \mathrm{V}$ at $50 \mathrm{MHz}$. After the cross calibration, the dispersion obtained over the whole SA array is smaller than the RMS of one antenna by more than one order of magnitude.

\section{Selection of radio events}

Most of the events detected by CODALEMA are anthropic, static and almost stationary (coming from sources giving accumulations in their directions of arrival). Several rejection algorithms are used online in the CODALEMA central DAQ to significantly suppress the parasitic events. 

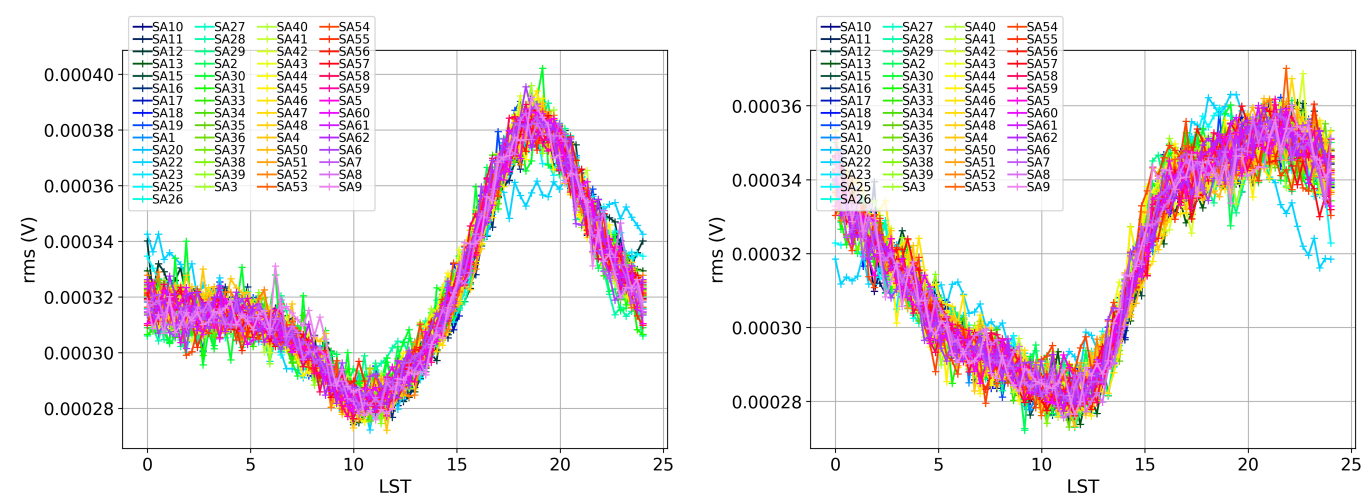

Figure 4: Results obtained after the cross calibration of the Butterfly antenna, at $50 \mathrm{MHz}$ and for an arbitrary month, for the EW (left) and NS (right) polarizations.

However, a lot of parasitic noises remain in the recorded events since the rejection algorithm rate was set to reach satisfactory number of events written on disk. Different methods have been developped and tested offline to improve the selection efficiency. They were not implemented online since the current CODALEMA duty cycle is mostly constrained by the T1 analog level efficiency and over simplicity. The different noise rejection algorithms applied offline on the batch of recorded events are based on:

- The rise time: the radio transients linked to a cosmic ray event have a rise time of the order of ten nanoseconds, making it possible to discriminate them from the radio transients linked to a parasitic noise transient which has a much greater rise time (a few tens to a hundred nanoseconds) and can exhibit several pulses during a short duration.

- The compacity: a shower event presents a regular pattern at ground, meaning that there should be no missing radio antennas in the pattern at ground close to the shower axis. This is not the case for a fortuitous event, where the pattern at ground can be irregular, with missing antennas (the high repetition rate of most noise events lead to high, but not necessarily synchronous occupation of the SA).

- The signal polarization: the angle of polarization gives information on the relative contribution of the two mechanisms at the origin of the radio emission, and thus can be compared to the predicted angle of polarization to give indications on the origin of the event (cosmic rays, fortuitous, presence of abnormal atmospheric electric field).

The performances of these algorithms have been tested on a large set of data have been tested, corresponding to $1,704,838$ events recorded by the central DAQ. Only 701 events remain after applying the algorithms, corresponding to $0.04 \%$ of the initial batch of events. Additional algorithms are considered in order to further reduce the number of parasitic transients from the overall set. We have checked that all the detected cosmic ray events (58) are present after the application of the noise rejection algorithms, corresponding roughly to 1.7 events per day. In the energy range of CODALEMA $\left(\left[10^{17}-10^{18}\right] \mathrm{eV}\right), \sim 5.1$ events per day are expected, which leads to an estimation of the efficiency for this batch of events of about $33 \%$. It is now necessary to compare these 1.7 events per day with the number of events in the energy range of CODALEMA giving an electric field greater than the detection threshold of the SA. This work is in progress. 


\section{Analysis method for data/simulations comparisons}

For each detected shower, we reconstruct the direction of arrival (DoA) using a plane fit. Using SELFAS3, a set of simulations is produced (40 protons, 10 iron showers) on a virtual antenna array at an arbitrary energy $\left(10^{17} \mathrm{eV}\right)$ with the corresponding DoA. Then for different shower core positions, we compare the measured values of the electric field to the simulated electric field scaled by a given factor to correct from the arbitrary energy. The best agreement is obtained by minimising the chi-squared (see [13] for more details). Exploiting the capabilities of the CODALEMA instruments, the reconstruction has been generalized by taking into account the high frequency data leading to a reconstruction in the EMF band which is very useful for inclined showers. It has been further generalized by decoupling the polarizations in the procedure. The quadratic summation of both polarizations EW and NS initially used implied a loss of information about each polarization and about the polarity of the signal. Finally, the reconstruction takes into account the information from the Compact Array leading to an hybrid reconstruction. To calculate the errors on the estimation of the shower parameters, a propagation of the errors is made. At each step of the comparison, an error on the measured values of the electric field is randomly calculated within the Gaussian distribution of the electric field values. This error is added to the electric field values, and the comparison procedure is repeated. After the propagation of the errors, the estimated parameter distributions of the shower is obtained. At the end of the analysis chain, the typical accuracies obtained have been evaluated to $15 \mathrm{~m}$ on the core position, $20 \%$ on the primary energy and $20 \mathrm{~g} \cdot \mathrm{cm}^{-2}$ on the atmospheric depth of the maximum of the shower development (noted $X_{\max }$ ).

\section{Cosmic Ray mass composition using radio signal with CODALEMA}

This method is applied to a data set composed of 246 events detected in coincidence between the SA and the particle detector array of CODALEMA. No cuts on the zenith angle or on the quality of the radio reconstruction are applied. Only a cut on the quality of the reconstruction of the event recorded by the particle detector array is applied (chi-square value). Figures 5 present the correlation between the primary cosmic ray energy estimated with the radio method and with the particle detectors method (left). The same correlation has been built between the energy estimated from the radio signal and the energy deduced from the particle detectors but using in both cases the core location given by the radio reconstruction (right). The estimated energy from the particle detectors are mostly lower than the estimated energy from the radio method. This is due to the fact that the surface covered by the autonomous station array is much larger than the one of the particle detector array. Thereby, most of the events falls outside the particle detector array, meaning that the estimated shower core positions are not reliable (the shower core position is pulled inside the particle detector array limits) and that the energies are underestimated. Using the core position estimated with the radio method, the energy from the particle detectors can be again estimated, and the correlation is clearly improved. This result indicates that the energy estimated with the radio method is reliable. In a further step, the cut made on the reconstruction of the scintillators will be removed, allowing a pure radio study of the mass dependence of the cosmic rays with energy, increasing the batch of events to $\sim 400$ events. This work is in progress.

A $X_{\max }$ estimate is also deduced for each event. The data are distributed in 7 energy bins, presented in figure 6 and compared to the mass estimated at the Pierre Auger Observatory, LOFAR, Hires/MIA, Yakutsk and Tunka. 

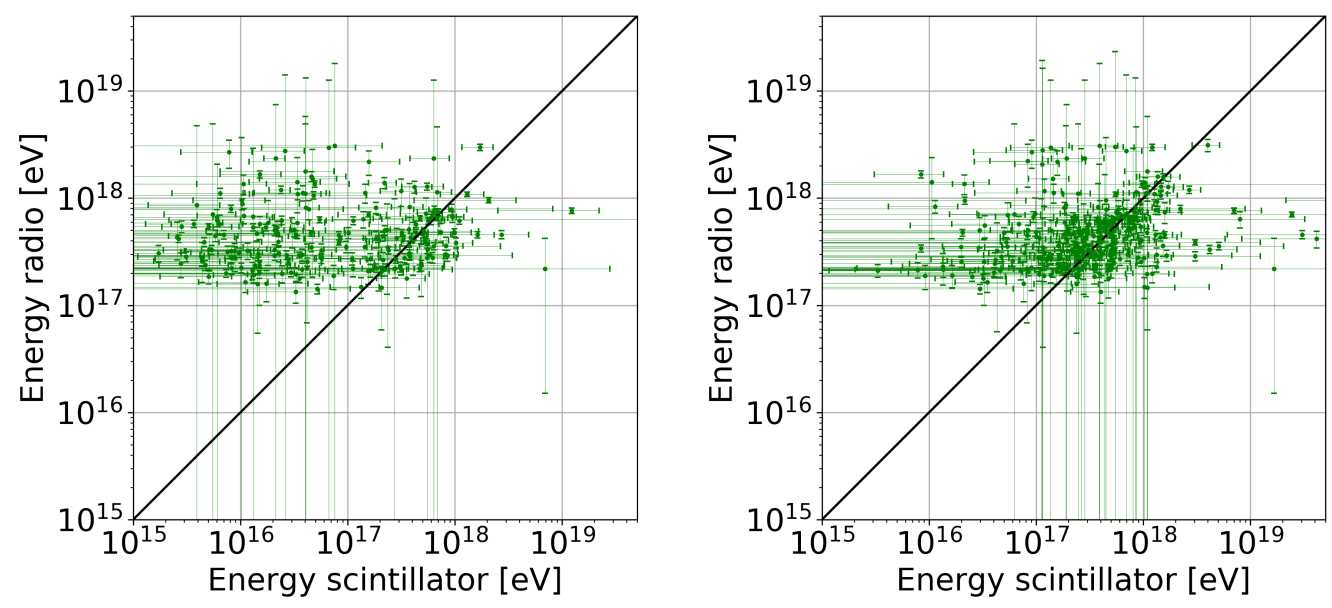

Figure 5: Left: correlation between the estimated primary cosmic ray energy with the radio method and with the particle detectors method. Right: correlation between the estimated primary cosmic ray energy with the radio method and with the particle detectors method with the core location taken from the radio signal analysis. The straight line accounts for a one-to-one correlation.

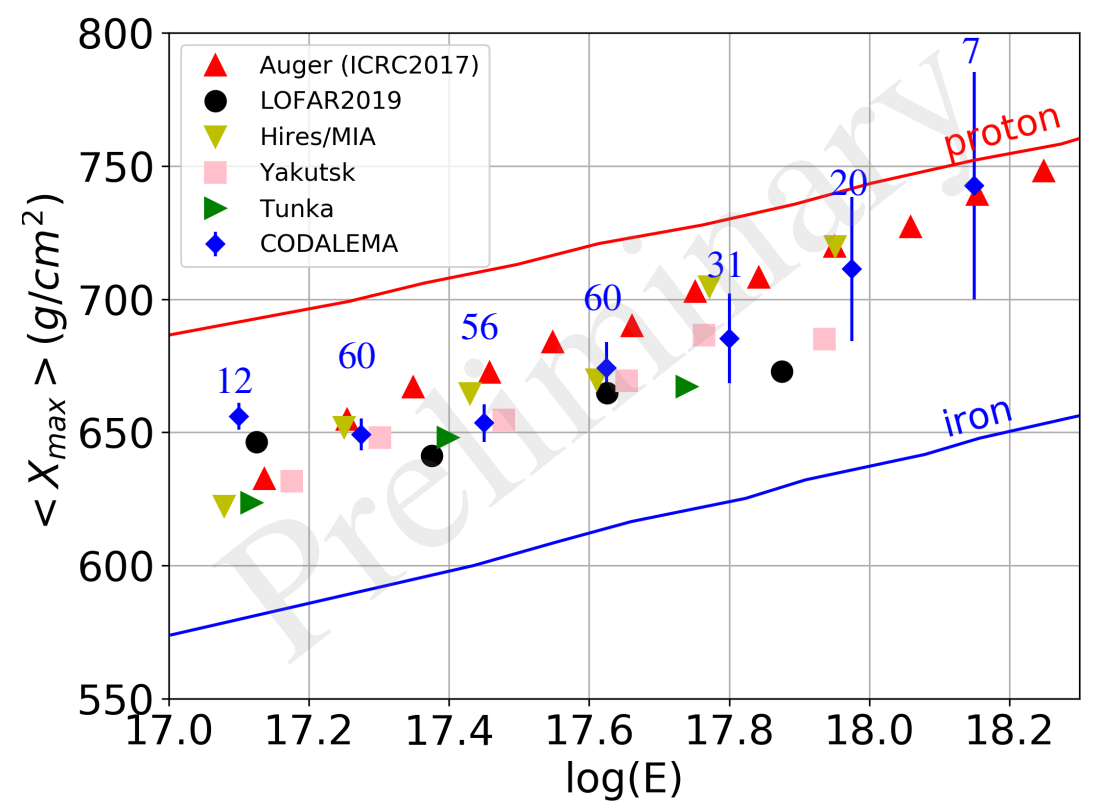

Figure 6: $X_{\max }$ mean values as a function of the energy of the primary by energy bins. The red triangles are the mean $X_{\max }$ measurements at the Pierre Auger Observatory, black points at LOFAR, inverted yellow triangles at Hires/MIA, pink squared at Yakutsk, green rotated triangles at Tunka and the blue diamonds are the results obtained with the method presented in this proceedings. Error bars include statistical uncertainties.

The seven energy bins are respectively composed of 12, 60, 56, 60, 31, 20 and 7 events. The reconstructed values are in very good agreement with the measurements of the other experiments, and are compatible with a light composition for energy above $10^{17.8} \mathrm{eV}$. 


\section{Conclusion}

CODALEMA/EXTASIS is a multi-wavelength radio experiment observing cosmic-ray induced air-showers in $[1-200] \mathrm{MHz}$. Its unique capabilities permit to better constrain the reconstruction of the cosmic ray properties, and to demonstrate for the first time that the study of atmospheric showers is possible over a very large frequency band in a continuous way, making it possible to scan different zones and processes of the air shower development and thus offering a more complete and rich view. However, due to the low efficiency of detection in the LF band, the most interesting frequency band for detecting air showers remains the $[20-200] \mathrm{MHz}$ band. Indeed, a low number of LF events has been detected over almost 2 years and moreover a strong correlation with the atmospheric electric field has been observed. The SDP has not yet been detected, sites higher in altitude are favored to optimize the chances of detection. The preliminary results on the composition of cosmic rays proposed by CODALEMA is in agreement with the other experiments such as Auger.

\section{Acknowledgements}

We thank the Région Pays de la Loire for its financial support of the Astroparticle group of Subatech and in particular for its contribution to the EXTASIS experiment, and the PNHE (Programme National Hautes Energies) from the french institutes IN2P3 and INSU for having also always supported the CODALEMA experiment, both financially and scientifically.

\section{References}

[1] F. D. Kahn and I. Lerche. Proceedings of the Royal Society of London A: Mathematical, Physical and Engineering Sciences, 289(1417):206-213, 1966.

[2] GA Askaryan. J. Exp. Theor. Phys. 21 (1962) 658.

[3] A. Bellétoile, R. Dallier, A. Lecacheux et al. Astroparticle Physics, 69:50-60, September 2015.

[4] D. Ardouin, A. Belletoile, C. Berat et al. Astroparticle Physics, 31:192-200, April 2009.

[5] Alexander Aab et al. Nuclear Instruments and Methods, A798:172-213, 2015.

[6] A. Nelles, S. Buitink, A. Corstanje et al. In American Institute of Physics Conference Series, volume 1535 of American Institute of Physics Conference Series, pages 105-110, May 2013.

[7] D. Charrier, R. Dallier, Antony Escudie et al. Astroparticle Physics, 113:6 - 21, 2019.

[8] Vincent Marin and Benoît Revenu. Astroparticle Physics, 35(11):733 - 741, 2012.

[9] F. Gaté, B. Revenu, D. García-Fernández et al. Astroparticle Physics, 98:38 - 51, 2018.

[10] Daniel García-Fernández, Benoît Revenu, Didier Charrier et al. Physical Review D, D97(10):103010, 2018.

[11] H. R. Allan et al V. B. Atrashkevich, O. V. Vedeneev. Soviet Journal of Nuclear Physics, 28:366, 1978.

[12] Angelica de Oliveira-Costa, Max Tegmark, B. M. Gaensler et al. Monthly Notices of the Royal Astronomical Society, 388:247, 2008.

[13] L. Martin, R. Dallier, A. Escudie et al. In International Cosmic Ray Conference, Bexco, Busan, South Korea, July 2017. 